\title{
A Review: Spectral Efficiency Improvement Techniques
}

\author{
Channaveeramma $\mathrm{E}^{1}$ \\ ${ }^{1}$ Assistant Professor of ECE Department, \\ Navodaya Institute of Technology, Raichur, \\ Karnataka, India.
}

\author{
Dr. K. M. Palaniswamy ${ }^{2}$ \\ ${ }^{2}$ Professor of ECE Department, \\ Dr. T. Thimmaiah Institute of Technology \\ Oorgaum, Kolar Gold fields, Karnataka, India.
}

\begin{abstract}
Wireless communications has become one of the fastest growing areas in our modern life and creates enormous impact on nearly every feature of our daily life. In this paper, I briefed out survey on Spectral efficiency improvement techniques which can satisfy the demands of future wireless gadgets.
\end{abstract}

Key Words: Spectral efficiency, LDPC, NOMA, IM,SM, OFDM,

\section{INTRODUCTION}

With the invention of wide range of wireless devices such as smart phones, tablets, etc, which are capable of highspeed multimedia streaming, wireless data traffic has increased dramatically [1],[2]. Spectral efficiency, the average data rate per unit bandwidth becomes highly important. For example, in Spain, it is expected to bid 1.5 billion EUR on a total of 310 $\mathrm{MHz}$ in $4 \mathrm{G}$ frequency bands [3]. With this high rate of data traffic, congestion problems will definitely bother the wireless networks in the near future [4]. Therefore, improving bandwidth efficiency or spectral efficiency becomes essential for future wireless communication systems.

The type of Channel coding technique, modulation techniques and the multiple access technique selected plays important role in improving spectral efficiency. Essentially the high spectral efficiency is achieved when higher modulation order and lower code rate is selected, leading to the need of elevated performance error correcting code.

Channel coding started with the Claude Shannon's 1948 landmark paper [5]. The next few decades, the researchers concentrated on the development of practical coding schemes that could approach channel capacity defined by the Shannon on AWGN channels. But this goal was challenging and impossible until the development of Turbo codes and LDPC codes.

Turbo codes are also identified as the parallelconcatenated convolutional codes (PCCC). These codes avoid the confusion of structure and randomness through concatenation and interleaving respectively. Turbo codes provides remarkable efficiency in AWGN and flat-fading channels for moderately low BER where as it suffers from increased latency, poor performance at very low BER.

Low density parity check coder allows parallelizable decoder, more amenable to high code rates, possess lower error rate, superior performance in bursts without Interleavers. Single LDPC can work better over collection of channels. The never ending demands of wireless communication are high speed, more data transfer, Low signal to noise ratio, low bit error rate, efficient bandwidth utilization, and efficient error correction and detection techniques. This paper reviews various error correcting codes that can lead to get the improved Spectral efficiency and Bit Error Rate for a communication system.

\subsection{SPECTRAL EFFICIENCY}

Spectral efficiency or bandwidth efficiency refers to the amount of information that can be transmitted over a given bandwidth in a specific communication system.

The three main areas where spectral efficiency can be improved is with efficient channel coding techniques, bandwidth efficient modulation techniques and massive multiple access techniques. The fourth effort to improve the spectral efficiency is combining channel coding, modulation and multiple access techniques.

\section{LITERATURE SURVEY}

The research work carried out by K.M.Palaniswamy[6] shows that the Adaptive modulation based MC-CDMA system or OFDMA system includes Turbo encoder in Rayleigh fading environment analyzed the BER performance for M-ary PSK, M-ary QAM, M-Ary MHPM and $\mathrm{M}$-ary CPM for BDBP (Bit Duration Bandwidth product) at $60 \mathrm{ksymbols} / \mathrm{sec}$. It is found that M-ary MHPM has achieved very low BER of upto $10^{-7}$ due to Adaptive Modulation. The Turbo coder with $1 / 3$ rating makes the system to work efficiently in adhoc environment at a constant BER concluding that MHPM is the best adaptive modulation system for both MC-CDMA and OFDMA accessing techniques.

HalaM.A.Mansour [7] presents analysis on the performance of various concatenated coding schemes such as serial concatenated convolutional code(SCCC), Parallel concatenated convolutional code(PCCC) and Parallel-serial concatenated convolutional code(P-SCCC) with M-ary PSK, M-ary QAM, FSK modulation techniques through AWGN channel. It is found that P-SCCC has better BER and SNR performance compared to PCCC and SCCC. Also P-SCCCMSK combination of coding and modulation scheme performs better compared to P-SCCC-PSK and P-SCCC-QAM.

The Adaptive modulation and Rate Coding[8] MultiCarrier Code Division Multiple Access(AMRC-MC-CDMA) technique proposed by T Jaya[3] using BPSK,QPSK amd Mary PSK modulation schemes in AWGN channel compares the BER performance at various code rates and found that AMRC- 
MC-CDMA method gives most favorable performance than the conventional MC-CDMA system used in fourth generation.

The research work carried out by Michael Yang[9] presents a new class of irregular Low Density Parity Check(LDPC) codes of moderate length $\left(10^{3} \leq \mathrm{n} \leq 10^{4}\right)$ and high rate $(\mathrm{R} \geq 3 / 4)$. It is found that this class of LDPC codes is same as class of systematic serial turbo codes and is the extension of irregular repeat-accumulate codes. A code design algorithm based on the combination of density evolution and differential evolution optimization with a modified cost function is presented which generate the error rate of $10^{-9}$ for moderate length, high-rate codes and it can be extended for other length and other error rates. Research towards this class of irregular LDPC promised to get application areas like magnetic and optical data storage where BER of $10^{-15}$ is often quoted and optical communication where error rates below $10^{-}$ ${ }^{10}$ is often quoted.

Arjan Meijerink[10] briefs study on M-ary (D)PSK modulation in coherence multiplex system shows that coherence multiplexed M-ary (D)PSK modulated signals can be demodulated by using either a phase synchronous receiver or a phase diversity receiver. Both these methods show the same decreasing network capabilities with increasing $\mathrm{M}$ in a coherence multiplex system with negligible dispersion and high received power. It points out that, increased $M$ increases symbol rate reduces the modulation speed and the clock frequency of the detection electronics and less susceptible for fiber dispersion. But receiver's complexity increases with increasing $\mathrm{M}$ and high accuracy and phase synchronization that of modulator is required.

S.Zhang and P.Y.Kam[11] evaluates the performance of coherent optical M-ary PSK/QAM using decision aided maximum likelihood phase estimation and obtains BER expressions of 16PSK and 16QAM analytically in the presence of a phase error and performance penalty is analyzed as a function of the phase error variance and is extended to M-ary PSK and 16QAM and predict the laser line width tolerance in different modulation formats and found that 16 QAM performs better for high spectral efficiency coherent optical communications.

Sakshi Gupta et.al [12] analyzes the performance of M-QAM and M-QPSK modulation schemes in AWGN and Multipath fading channel in terms of BER v/s SNR. It is found that performance of WCDMA system in AWGN channel with QPSK modulation is better compared to that of 16QAM. Also QPSK shows better performance compared to 16QAM in AWGN and multipath Rayleigh fading channel. 16 QAM scheme suffers from adjacent carrier interference producing abnormal values of BER and hence degrading WCDMA performance.

De Souza,S Lemos-Neto2 and Valdemar C da Rocha[13], employs a bimode decoder for LDPC codes for the AWGN channel. The oscillating behavior of the error rate versus number of iterations, featured by some LDPC codes of the IEEE802.11n standard, was exploited in order to take advantage of the so-called valley points in the error event analysis. Simulation results show that a more efficient use of the HD system is obtained when the min-sum BP decoder employs a maximum number of iterations corresponding to points close to and possibly including a valley point and avoiding values close to and possibly including a peak point. The goal achieved here was an enhancement of the performance of the bi-modal hybrid decoder available.

Gorden.L[14],explores various physical layer research challenges in MIMO-OFDM system design, including physical channel measurements and modeling, analog beam forming techniques using adaptive antenna arrays, space-time techniques for MIMO-OFDM, error control coding techniques, OFDM preamble and packet design, and signal processing algorithms used for performing time and frequency synchronization, channel estimation, and channel tracking in MIMO-OFDM systems. Finally, the paper considers a software radio implementation of MIMO-OFDM.

Nauman F. Kiyani, Umar H. Rizvi, Jos H. Weber and Gerard J. M. Janssen[15], presents signal space diversity for multi level modulation schemes with LDPC coding. It was found that LDPC coding with proper constellation rotation leads to a significant performance improvement over the non rotated case in a system using signal space diversity over uncorrelated Rayleigh fading channels.

Jiancun Fan1, Shengjie Guo2, Xiangwei Zhou2, Yajie Ren1, Geoffrey Ye Li3, And Xi Chen4[16], provides an overview on faster than Nyquist, transmitter and receiver optimization, pulse shaping and transmitter hardware implementation, viterbi and BCJR algorithms and future challenges in the corresponding areas.

Erdal Arıkan, Najeeb ul Hassan, Michael Lentmaier, Guido Montorsi and Jossy Sayir[17] lists the challenges and new directions in channel coding. It presents 3 major research areas as Spatially coupled LDPC codes, non binary LDPC codes and Polar codes.

Zhang Zhi, Qi Binghua, Zhang Ping[18], approached a method to combine the LDPC codes with modulation schemes having better spectral efficiency in turn to increase the spectral efficiency of communication. The technique was implemented on BPSK and QPSK modulation schemes.

Stefan Nowak, Grzegorz Smietanka and Ruediger Kays[19], shows that application of non binary LDPC codes in combination with symbol mapping and higher order constellations can lead to significant gains in terms of BER and PER. It employs partial symbol mapping for reducing the complexity and retaining much of the good performance with higher code rates. Simulation results for 16, 64 and 256 QAM have been compared.

Hongxin Song and J. R. Cruz[20]concentrates on developing the low complexity LDPC decoding algorithms. LLR-BP decoding were described, starting with the most commonly used BP-based approximation of the check-node update. 
Adrian Voicila, David Declercq, François Verdier, Marc Fossorier, Pascal Urard[21], presents Extended minimum sum algorithms for non-binary LDPC codes. It analyzes EMS GF(64), BP GF(64), BP GF(256), EMSGF(64), EMSGF(256) decoding algorithms for achieving low complexity and low memory requirement for non-binary LDPC codes.

\section{METHODOLOGY}

The most commonly used parameters in digital communication system are Bit Error rate, signal to noise ratio, high data rate, efficient bandwidth utilization (Spectral efficiency), error correction techniques.

The most common coders used for channel coding are convolution coders, Turbo coders and Low Density Parity Check Codes. Convolutional codes are error detecting codes used to reliably transmit digital data over unreliable communication channel system. These are well suited for large data streams, more energy efficient than block codes and find their applications in areas like deep space communication, satellite and terrestrial digital video broadcasting. But their computational complexity increases exponentially with the length of the code.

The general Block Diagram of a wireless communication system is as follows.

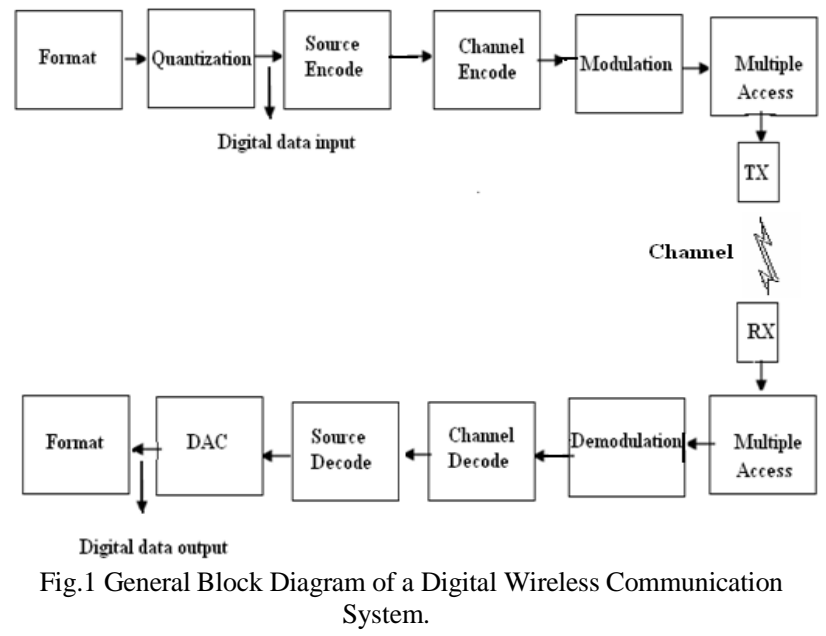

\subsection{Formatting, Source encoding and Decoding}

Formatting transforms the source information into digital symbols. The digital symbols are then encoded. The purpose of this is to compress the data in some manner to remove redundancy. For analog source signals; source coding performs an analog to digital conversion using any of the following. Character coding, sampling, quantization, PCM, Differential PCM, block coding. Decoding reverses the source coding. The different types of source coders are Bernoulli coder, Random Number generator etc.

\subsection{Channel Coding and Decoding}

Channel encoder and decoder are used at the transmitter and receiver respectively to introduce the redundancy to the stream of bits to enable error detection and correction. Commonly used coders are Block Codes, convolutional Coders and Turbo Coders, TCM, LDPC.

\subsection{Modulation and Demodulation}

Modulation is a process of varying any of the characteristics (Amplitude, Frequency, Phase) of a carrier wave based on the information or message or baseband signal in order to achieve multiple access, shifting the contents of a baseband signal to higher frequency whichin turn reduces the antenna size.

Demodulation is a reverse process of modulation which extracts the message signal back from the modulated signal. Digital demodulation is achieved with the aid of reference waveforms and is said to be coherent if the reference waveform contains phase information and non-coherent otherwise.

The different modulation schemes are PSK, BPSK, QPSK, 8PSK and so on, hence called as M-Ary PSK where $\mathrm{M}$ is variable.

\subsection{Multiple Access Techniques.}

The multiple access stage defines how signal from different sources are combined and have access to the channel. Typical multiple access schemes are TDMA, FDMA, CDMA, CSMA, OFDMA, MC-CDMA.

\subsection{Transmission/Reception:}

The wireless networks operate at RF frequencies, so up conversion to these frequencies and amplification of signals before transmission with a suitable antenna is also necessary. Reception involves amplification and down conversion to an IF frequency.

\subsection{Quantization and Digital to Analog Converters:}

Quantization and Digital to analog Converters are used to convert the incoming analog signal to digital at the transmitter and vice versa at the receiver.

\subsection{Types of Channels:}

The typical wireless channels are Rayleigh fading channel, Rician channel, Nakagami channel. In M-ary PSK, as the value of $\mathrm{M}$ increases, the spectral efficiency increases. With the use of Non binary LDPC codes, we can get higher spectral efficiency as earlier research [14] shows for QAM. MPSK and LDPC combinations have been used only for $\mathrm{M}=2$ and $\mathrm{M}=4$ and $\mathrm{M}=8$ [11]. Here, we continue MPSK-LDPC combination for greater values of $\mathrm{M}$ and hence increasing the spectral efficiency to meet the demands of future wireless Communication.

\section{MODULATION TECHNIQUES}

The different modulation techniques are ASK,FSK,PSK out of which PSK was considered efficient since it carries information in terms of Phase with constant amplitude. Various studies have been done with for M-PSK and M-QAM and found that M-QAM gives better performance in terms of BER and SE when the M values increases beyond 8 . The next family of modulation schemes was introduced called index modulation[22] which sends the information not only in terms of basic parameters amplitude, frequency and phase but also map information bits by altering the on/off status of their transmission entities. Modulation types, precoder matrices, 
spreading codes, time slots signal powers, dispersion matrices, loads and so on.

As the demand for spectral efficiency and energy efficiency increased in 5G gadgets operation, the variation of IM called spatial modulation which used the indices of the transmit antennas of MIMO system for sending additional information[23]. The SM finds its application in Full-Duplex Networks, Cooperative networks, Cognitive Radio Networks, Massive MIMO and Multi-User Systems. Generalized SM, Quadrature SM, Enhanced SM, Differential SM, Optical SM, RECONFIGURABLE ANTENNA-BASED INDEX modulation techniques, Channel modulation, Space-Time Channel Modulation, OFDM-IM, OFDM In-Phase/Quadrature are the different spectral efficient modulation techniques [24]. The Fully-Generalised Spatial Modulation System, The FullyQuadrature Spatial Modulation models have been proposed in [26] which promise to overcome the disadvantages of SM and improves the spectral efficiency and BER performance for future wireless communication.

\section{MULTIPLE ACCESS TECHNIQUES}

The various modulation techniques and multiple access techniques to support $5 \mathrm{G}$ networks. The requirements of $5 \mathrm{G}$ networks such as High spectral efficiency, loose synchronization, flexibility are satisfied by the modulation techniques based on different principles, like pulse shaping, precoding, subband filtering, GI shortening, and mapping of symbols in the delay-Doppler domain. Filter Bank Multicarrier (FBMC), Generalized Frequency Division Multiplexing (GFDM), pulse shaped OFDM and QAM-FBMC are the techniques based on pulse shaping which contribute to reduce OOB leakage. Subband filtering is another technique which help reduce OOB leakage. Universal filtered multicarrier(UFMC) and filtered OFDM(f-OFDM) are the two typical modulation techniques. GI discrete Fourier transform spread OFDM (GI DFT-s-OFDM), spectrally-precoded OFDM

Fig.2.Classification of multiplexing techniques.

(SP-OFDM), and orthogonal time frequency and space (OTFS) are the other modulation techniques helping to reduce OOB

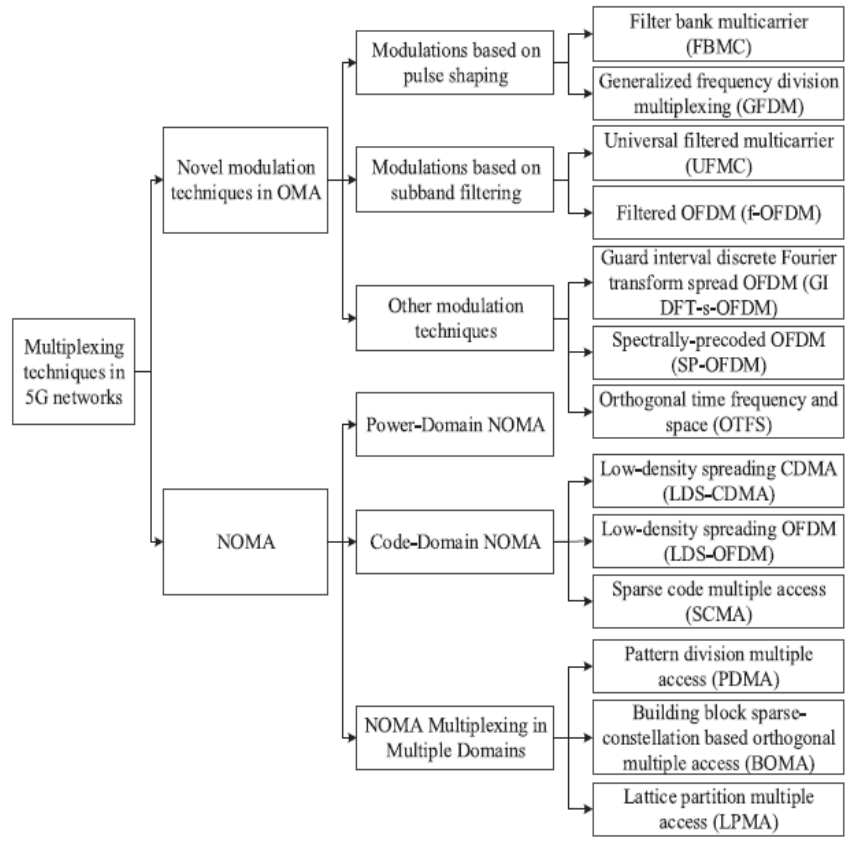

leakage. Performance comparison of above modulation techniques is done with respect to PSD and BER. OTFS was found to give good performance[25].

\section{CONCLUSION}

The spectral efficiency requirement of future wireless communication finds its lights in channel coding techniques such as LDPC codes, Polar codes, modulation techniques such as spatial F-GSM, F-QSM, and multiple access techniques such as variations of Non orthogonal multiple access. Coded modulation techniques and combining them with multiple access techniques also aid in improving the performance as spectral efficiency and BER.

\section{REFERENCES}

[1] J. Qiao, X. Shen, J. Mark, Q. Shen, Y. He, and L. Lei, “'Enabling device to device communications in millimeter-wave $5 \mathrm{G}$ cellular networks," IEEE Commun. Mag., vol. 53, no. 1, pp. 209_215, Jan. 2015.

[2] E. Hossain and M. Hasan, " 5 G cellular: Key enabling technologies and research challenges," IEEE Instrum. Meas. Mag., vol. 18, no. 3, pp. 11_21, Jun. 2015.

[3] F. F. Lanas and P. P. Gomez, “4G mobile licenses under auction in Spain [Global communications newsletter]," IEEE Commun. Mag. vol. 49, no. 7, pp. 1_4, Jul. 2011.

[4] J. G. Andrews et al., "What will 5G be?" IEEE J. Sel. Areas Commun., vol. 32, no. 6, pp. 1065_1082, Jun. 2014.

[5] C. E. Shannon, BA mathematical theory of communication, [ Bell Syst. Tech. J., vol. 27, pp. 379-423 and 623-656, 1948.

[6] Kugalur Muthusamy Palaniswamy, Veerappa Gounder Palanisamy, The Optimistic Adaptive Modulation Technique For Future Wireless Communication, Journal of Computer Science, ISSN 1549-3636@ 2013 Science Publications.

[7] Hala M. A. Mansour, Labib Francis Gergis, Mostafa A. R. Eltokhy, Hoda Z. Said, Performance Analysis for Concatenated Coding schemes with Efficient Modulation Techniques International Journal of Soft Computing and Engineering (IJSCE) ISSN: 22312307, Volume-2, Issue-1, March 2012.

[8] T. Jaya And E. Gopinathan, Adaptive Modulation And Rate Coding Multi Carrier Code Division Multiple Access Technique for $4 \mathrm{G}$ Mobile Communication Systems, ARPN Journal of Engineering and Applied Sciences, Vol. 10, No. 13, July 2015.

[9] Michael Yang, Student Member, IEEE, William E. Ryan, Senior Member, IEEE, and Yan Li, Student Member, IEEE,Design of Efficiently Encodable Moderate-Length High-Rate Irregular LDPC Codes, IEEE Transactions On Communications, Vol. 52, No. 4, April 2004.

[10] Arjan Meijerink, Geert Heideman and WimvnEtten, M-ary (D)PSK modulation in coherence multiplex systems, University of Twente, Department of Electrical Engineering, Telecommunication Engineering Group, Netherlands.

[11] S. Zhang, P. Y. Kam, J. Chen, and C. Yu, Bit-error rate performance of coherent optical M-ary PSK/QAM using decisionaided maximum likelihood phase estimation, Department of Electrical \& Computer Engineering, National University of Singapore,117576, Singapore. 24 May 2010 / Vol. 18, No. 12 / OPTICS EXPRESS 12088.

[12] Performance Investigation for Different Modulation Techniques in WCDMA with Multipath Fading Channels, Sakshi Gupta, Himanshu Sharma, International Journal of Advanced Research in Computer Science and Software Engineering, Volume 2, Issue 7, July 2012

[13] Walter Prado de Souza Guimarães1*, José S Lemos-Neto2 and Valdemar C da Rocha Jr2, "Efficient use of a hybrid decoding technique for LDPC codes", Guimarães et al. EURASIP Journal on Wireless Communications and Networking 2014, 2014:32

[14] Gordon L. Stüber, Fellow, IEEE, John R. Barry, Member, IEEE,Steve W.Mclaughlin, Senior Member, IEEE, Ye (Geoffrey) Li, Senior Member, IEEE,Mary Ann Ingram, Senior Member, IEEE 
And Thomas G. Pratt, Member, IEEE, Broadband MIMO-OFDM WirelessCommunications, (C) 2004 IEEE.

[15] Nauman F. Kiyani, Umar H. Rizvi, Jos H. Weber and Gerard J. M. Janssen, "Optimized Rotations for LDPC-coded MPSK Constellations with Signal Space Diversity", Proceedings of WCNC 2007, IEEE Communications Society.

[16] Jiancun Fan1, Shengjie Guo2, Xiangwei Zhou2, Yajie Ren1, Geoffrey Ye Li3, And Xi Chen4, "Faster-Than-Nyquist Signaling: An Overview", Received November 21, 2016, accepted December 22, 2016, date of publication February 7, 2017, date of current version March 13, 2017. Digital Object Identifier 10.1109/ACCESS.2017.2657599

[17] Erdal Arkkan, Najeeb ul Hassan, Michael Lentmaier, Guido Montorsi and Jossy Sayir "Challenges and some new directions in channel coding" Journal Of Communications And Networks, VOL. 17, NO. 4, August 2015.

[18] Zhang Zhi, Qi Binghua, Zhang Ping, "Low-Density Parity-Check Codes and High Spectral Efficiency Modulation" The $\mathbf{1 4}^{\text {th }}$ IEEE 2003 International Symposium on Persona1,Indoor and Mobile Radio Communication Proceedings.

[19] Stefan Nowak, Grzegorz Smietanka and Ruediger Kays, "High Efficiency Broadband Transmission with LDPC Codes over GF(2s)", 2012

[20] Hongxin Song, Member, IEEE, and J. R. Cruz, Fellow, IEEE, "Reduced-Complexity Decoding of Q-ary LDPC Codes for Magnetic Recording”, IEEE Transactions On Magnetics, Vol. 39, No. 2, March 2003.

[21] Adrian Voicila, David Declercq, François Verdier, Marc Fossorier, Pascal Urard, "Low-complexity, Low-memory EMS algorithm for non-binary LDPC codes", 2007.

[22] E. Basar, "Index modulation techniques for $5 \mathrm{G}$ wireless networks,"IEEE Commun. Mag., vol. 54, no. 7, pp. 168-175, June 2016.

[23] R. Mesleh, H. Haas, S. Sinanovic, C. W. Ahn, and S. Yun, "Spatial modulation,” IEEE Trans. Veh. Technol., vol. 57, no. 4, pp. 2228 2241,July 2008

[24] Ertugrul Basar," Index Modulation Techniques for NextGeneration Wireless Networks"', IEEE ACCESS, VOL. 5, AUGUST 2017.

[25] Yunlong Cai, Senior Member, IEEE, Zhijin Qin , Member, IEEE, Fangyu Cui, "Modulation and Multiple Access for 5G Networks", IEEE Communications Surveys \& Tutorials, VOL. 20, No. 1, First Quarter 2018

[26] HANY S. HUSSEIN,"'Spectral Efficient Spatial Modulation Techniques", IEEE Access, Digital Object Identifier 10.1109/ACCESS.2018.2885826. 